archives

of thermodynamics

Vol. 35(2014), No. 4, 29-42

DOI: $10.2478 /$ aoter-2014-0031

\title{
Analytical solutions of heat transfer for laminar flow in rectangular channels
}

\author{
WITOLD RYBIŃSKI ${ }^{1}$ \\ JAROSŁAW MIKIELEWICZ
}

The Szewalski Institute of Fluid-Flow Machinery, Polish Academy of Sciences, Fiszera 14, 80-231 Gdańsk, Poland

\begin{abstract}
The paper presents two analytical solutions namely for Fanning friction factor and for Nusselt number of fully developed laminar fluid flow in straight mini channels with rectangular cross-section. This type of channels is common in mini- and microchannel heat exchangers. Analytical formulae, both for velocity and temperature profiles, were obtained in the explicit form of two terms. The first term is an asymptotic solution of laminar flow between parallel plates. The second one is a rapidly convergent series. This series becomes zero as the cross-section aspect ratio goes to infinity. This clear mathematical form is also inherited by the formulae for friction factor and Nusselt number. As the boundary conditions for velocity and temperature profiles no-slip and peripherally constant temperature with axially constant heat flux were assumed (H1 type). The velocity profile is assumed to be independent of the temperature profile. The assumption of constant temperature at the channel's perimeter is related to the asymptotic case of channel's wall thermal resistance: infinite in the axial direction and zero in the peripheral one. It represents typical conditions in a minichannel heat exchanger made of metal.
\end{abstract}

Keywords: Analytical solution; Heat transfer; Rectangular channel; Laminar flow; Fully developed

\footnotetext{
${ }^{1}$ Corresponding Author. E-mail: witold.rybinski@imp.gda.pl
} 


\section{Nomenclature}

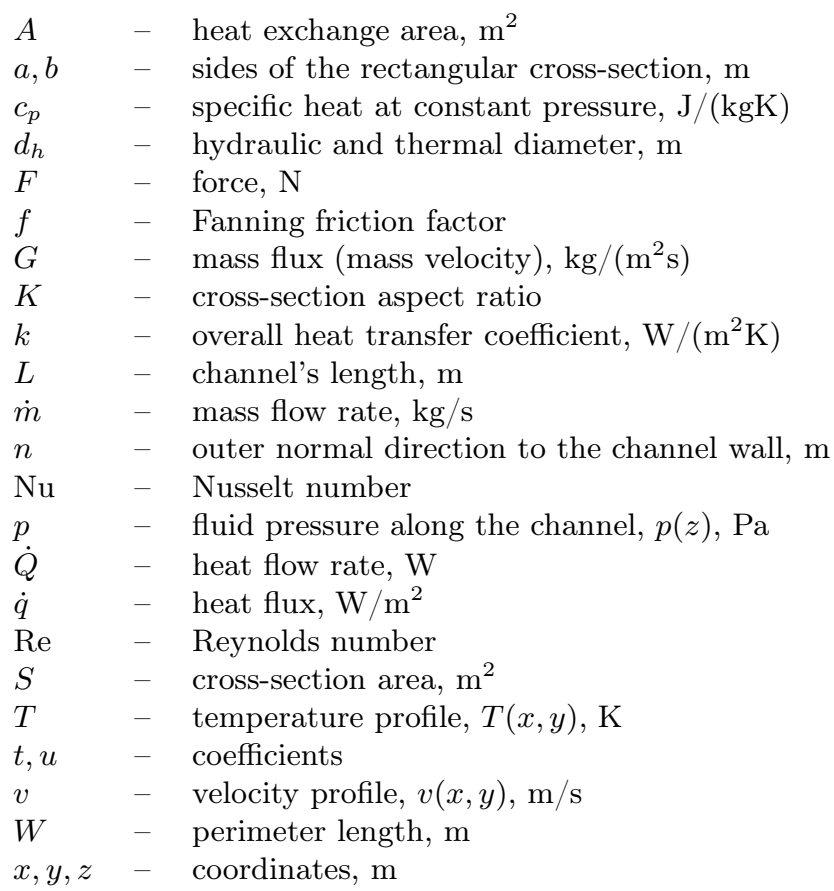

\section{Greek symbols}

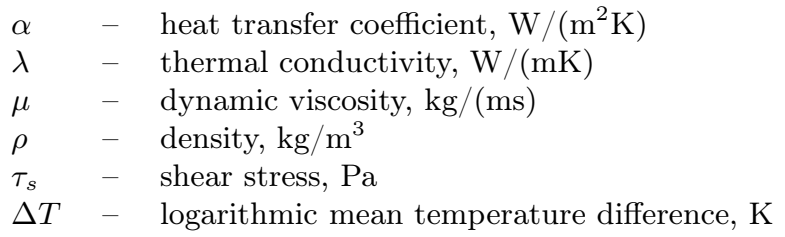

\section{Subscripts}

$\begin{array}{ll}c, h & - \text { cold and hot fluid } \\ \text { circle, square, plates } & - \text { cross-section types } \\ n & -n \text {-th term in a series }(n=1, \ldots, \infty) \\ w & -\quad \text { channel's wall } \\ - & -\quad \text { mean value over the cross-section or perimeter }\end{array}$

\section{Introduction}

Minichannel heat exchangers with laminar fluid flows are suitable for small scale organic Rankine cycle (ORC) and other applications like electronic equipment. One of the task which may help the design of minichannel heat 
exchangers is to find the analytical form of laminar fluid flow velocity and temperature profiles in a rectangular minichannel, Fanning friction factor and Nusselt number. It can be further applied in an analytical model of heat transfer in minichannel heat exchanger. Rectangular cross-section of minichannels is typical in this type of heat exchangers. The analytical solutions also help in testing numerical algorithms as the reference solutions. Both analytical and approximate formulae for velocity and temperature profiles, Fanning friction factor and Nusselt number exist in the literature (for example in [1]). However, these analytical solutions consist of double series or single series of not very fast convergence.

In the paper the analytical formulae for these quantities are presented. The solutions consist of two parts: the asymptotic solution of laminar flow between parallel plates and the rapidly convergent single series. This series becomes zero as the cross-section aspect ration goes to infinity. The numerical results are compared with the results of the utilized existing formulae.

The main goal in this paper is to obtain the fast convergent formula for Nusselt number. However, since it needs derivation of the fluid velocity profile, the formula for Fanning friction factor is also derived.

\section{General information about minichannel heat exchanger}

A minichannel heat exchanger consists of many double layers of rectangular channels in which hot and cold fluids flow. Schematic view of the minichannel heat exchanger and channel's geometry is shown in Fig. 1. The channel's cross-section is described by $x$ and $y$ coordinates (Fig. 2). The $z$ coordinate is an axis of the channel.

Total heat flow rate in the minichannel heat exchanger is given by the general formula

$$
\dot{Q}=k A \Delta T .
$$

If thermal resistance of channel walls is negligible, overall heat transfer coefficient is given by

$$
\frac{1}{k}=\frac{1}{\alpha_{h}}+\frac{1}{\alpha_{c}} .
$$

Heat transfer coefficient $\alpha$ of the fluid flow can be calculated from the definition of the Nusselt number

$$
\mathrm{Nu}=\frac{\alpha d_{h}}{\lambda}
$$


General view of the heat exchanger

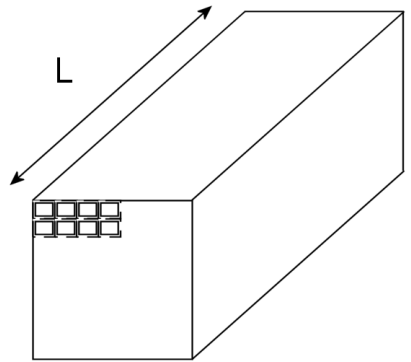

Channel's cross-section

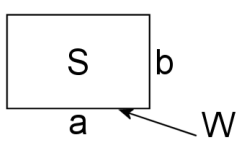

Figure 1: Schematic view of a minichannel heat exchanger.

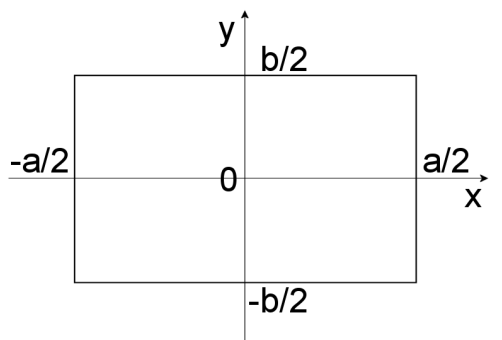

Figure 2: Coordinates of the channel's cross-section.

In order to derive the formula for Nusselt number, it is necessary to derive the formula for velocity profile of the laminar fluid flow first. This also gives a possibility to calculate Fanning friction factor and pressure drop along the channel.

Channel's geometry is described by its cross-section aspect ratio

$$
K=\frac{a}{b} .
$$

For square cross-section $K=1$, whereas for infinite wide plates $K \rightarrow \infty$.

It is assumed that hydraulic and thermal equivalent channel diameters are the same and are given by

$$
d_{h}=\frac{4 S}{W}=2 \frac{K}{K+1} b
$$

where

$$
S=a b, \quad W=2(a+b) .
$$

There are two extreme cases of the equivalent diameter (for square duct and for infinite wide plates):

$$
d_{\text {h.square }}=b ; \quad d_{\text {h.plates }}=2 b .
$$




\section{Derivation of the velocity profile and Fanning friction factor}

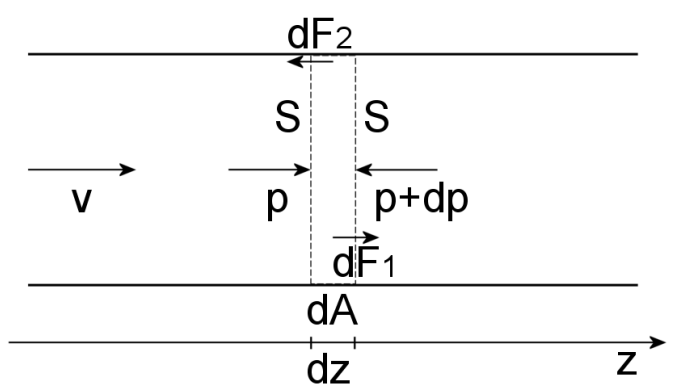

Figure 3: Momentum balance in the fluid element.

Two forces act on the fluid element (Fig. 3):

$$
d F_{1}=-S \frac{d p}{d z} d z, \quad d F_{2}=-\bar{\tau}_{s} d A,
$$

where mean wall shear stress, $\bar{\tau}_{s}$, and the wall surface element, $d A$, are given by

$$
\bar{\tau}_{s}=\frac{1}{W} \int_{W} \tau_{s} d W, \quad d A=W d z .
$$

The sum of $d F_{1}$ and $d F_{2}$ is equal zero for a steady state flow:

$$
d F_{1}+d F_{2}=0 .
$$

Use of $(9),(11)$ and the definition of $\mathrm{d}_{h}(6)$ gives

$$
\bar{\tau}_{s}=-\frac{d_{h}}{4} \frac{d p}{d z} .
$$

Fanning friction factor, $f$, is defined by the formula for mean wall shear stress

$$
\bar{\tau}_{s}=f \frac{\rho \bar{v}^{2}}{2},
$$

where the mean flow velocity is defined by

$$
\bar{v}=\frac{1}{S} \int_{S} v d S .
$$


Combination of (12) and (13) gives

$$
f=-\frac{1}{\rho \bar{v}^{2}} \frac{d_{h}}{2} \frac{d p}{d z} .
$$

Use of Reynolds number

$$
\text { Re }=\frac{G d_{h}}{\mu} \quad \text { where } \quad G=\frac{\dot{m}}{S}=\rho \bar{v},
$$

gives the formula for the Fanning friction factor

$$
f=-\frac{1}{\operatorname{Re} \bar{v}} \frac{d_{h}^{2}}{2} \frac{1}{\mu} \frac{d p}{d z} .
$$

The above general derivation independent of the cross-section shape is based on the same momentum balance as described in [2] where circular ducts are considered.

Calculation of $f$ demands the knowledge of fluid flow mean velocity, $\bar{v}$. For fully developed steady state incompressible laminar flow, the velocity profile, $v(x, y)$, is a solution of the Poisson equation

$$
\frac{\partial^{2} v}{\partial x^{2}}+\frac{\partial^{2} v}{\partial y^{2}}=\frac{1}{\mu} \frac{d p}{d z},
$$

with the Dirichlet boundary condition

$$
\left.v\right|_{w}=0 .
$$

Equation (17) was derived from Navier-Stokes equation under the assumption of constant physical fluid properties. This means velocity profile is independent of the temperature one. The solution of this nonuniform linear partial differential equation is a sum of two terms:

- particular solution of Poisson equation (in the form of simple expression) with the boundary condition for parallel plates,

- general solution of Laplace equation (in the form of cosine Fourier series) with the boundary condition equal to the difference between the condition (18) and the condition of the first term.

Velocity profile, $v(x, y)$, is then given by

$$
v(x, y)=\frac{1}{\mu} \frac{d p}{d z}\left[\frac{1}{2}\left(y^{2}-\frac{b^{2}}{4}\right)-4 b^{2} \sum_{n=1}^{\infty} \frac{(-1)^{n}}{u_{n}^{3}} \frac{\cosh \left(u_{n} K \frac{x}{a}\right)}{\cosh \left(u_{n} K \frac{1}{2}\right)} \cos \left(u_{n} \frac{y}{b}\right)\right],
$$


where

$$
u_{n}=(2 n-1) \pi .
$$

The Fourier series in (19) is convergent since the first term $(-1)^{n} / u_{n}^{3}$ is absolute convergent, the second one is bounded and less or equal to 1 for $-a / 2 \leq x \leq a / 2$, the third (cosine) term is always bounded.

Mean velocity is calculated by double integration

$$
\bar{v}=\frac{1}{a b} \int_{-a / 2}^{a / 2} \int_{-b / 2}^{b / 2} v(x, y) d x d y
$$

which gives the result

$$
\bar{v}=-\frac{1}{\mu} \frac{d p}{d z} \frac{d_{h}^{2}}{48}\left(\frac{K+1}{K}\right)^{2}\left[1-\frac{192}{K} \sum_{n=1}^{\infty} \frac{1}{u_{n}^{5}} \tanh \left(u_{n} K \frac{1}{2}\right)\right] .
$$

Combination of (16) and (22) gives the final formula for the Fanning friction factor

$$
f=\frac{24}{\operatorname{Re}} \frac{\left(\frac{K}{K+1}\right)^{2}}{1-\frac{192}{K} \sum_{n=1}^{\infty} \frac{1}{u_{n}^{5}} \tanh \left(u_{n} K \frac{1}{2}\right)} .
$$

The series appearing in (23) is rapidly convergent. In practice, for $1 \leq K \leq$ 20 achievement of the relative accuracy $10^{-6}$ demands use of maximum 9 first values.

\section{Derivation of the temperature profile and Nusselt number}

It is assumed that wall temperature $T_{w}=$ const at the perimeter. Heat flow dQ leaving a fluid element (Fig. 4) is equal to

$$
d \dot{Q}=\dot{m} c_{p} \bar{T}-\dot{m} c_{p}(\bar{T}+d \bar{T})=-\dot{m} c_{p} \frac{d \bar{T}}{d z} d z
$$

where the mean fluid temperature is given by

$$
\bar{T}=\frac{1}{S \bar{v}} \int_{S} v T d S .
$$




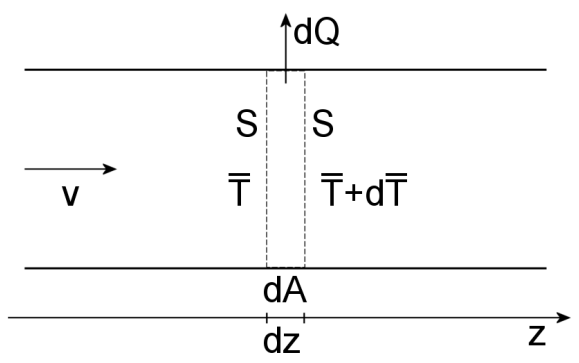

Figure 4: Energy flow balance in the fluid element.

Heat transfer coefficient, $\alpha$, is defined by Newton's law of cooling

$$
d \dot{Q}=\alpha\left(\bar{T}-T_{w}\right) d A=\alpha\left(\bar{T}-T_{w}\right) W d z .
$$

Combination of (24) and (26) gives

$$
-\dot{m} c_{p} \frac{d \bar{T}}{d z}=\alpha\left(\bar{T}-T_{w}\right) W .
$$

Use of the definition of $d_{h}(6)$ and the equation for mass flow rate

$$
\dot{m}=\rho \bar{v} S,
$$

gives

$$
\alpha=-\rho c_{p} \frac{d \bar{T}}{d z} \frac{d_{h}}{4} \frac{\bar{v}}{\bar{T}-T_{w}} .
$$

Combination of (6) and (29) gives the formula for Nusselt number

$$
\mathrm{Nu}=-\frac{\rho c_{p}}{\lambda} \frac{d \bar{T}}{d z} \frac{d_{h}^{2}}{4} \frac{\bar{v}}{\bar{T}-T_{w}} .
$$

The above general derivation, independent of the cross-section shape, is based on the same energy balance as described in [2] where circular ducts are considered.

Calculation of $\mathrm{Nu}$ number demands knowledge of fluid flow mean temperature, $\bar{T},(25)$. For fully developed steady state incompressible laminar flow, without internal heat sources and energy dissipation, the temperature profile, $T(x, y)$, is a solution of the Poisson equation

$$
\frac{\partial^{2} T}{\partial x^{2}}+\frac{\partial^{2} T}{\partial y^{2}}=\frac{\rho c_{p}}{\lambda} \frac{d \bar{T}}{d z} v
$$


with the Dirichlet boundary condition

$$
\left.T\right|_{w}=T_{w}=\text { const } .
$$

Equation (31) was derived from Fourier-Kirchhoff equation under assumption of constant axial wall heat flux, $\dot{q}_{w}$, (along $z$ axis) with uniform peripheral wall temperature $T_{w}$. This means wall thermal resistance is infinite in the axial direction and zero in the peripheral direction. This type of condition is known in the literature as $\mathrm{H} 1$ type, according to [1]. Constant physical fluid properties are also assumed as well as the lack of fluid axial heat conduction.

The solution of Eq. (31) was obtained by use of the finite Fourier transform with the same orthogonal set of functions as in the solution for velocity profile (19). This way, the temperature profile is a sum of two terms (simple expression and cosine Fourier series):

$$
\begin{gathered}
T(x, y)=T_{w}+\frac{\rho c_{p}}{\lambda} \frac{d \bar{T}}{d z} \frac{1}{\mu} \frac{d p}{d z}\left\{\frac{1}{8}\left(\frac{y^{4}}{3}-\frac{b^{2} y^{2}}{2}+\frac{5 b^{4}}{48}\right)+\right. \\
+2 b^{4} \sum_{n=1}^{\infty} \frac{(-1)^{n}}{u_{n}^{5}}\left[2+u_{n} K \frac{1}{2} \tanh \left(u_{n} K \frac{1}{2}\right)-u_{n} K \frac{x}{a} \tanh \left(u_{n} K \frac{x}{a}\right)\right] \times \\
\left.\times \frac{\cosh \left(u_{n} K \frac{x}{a}\right)}{\cosh \left(u_{n} K \frac{1}{2}\right)} \cos \left(u_{n} \frac{y}{b}\right)\right\}
\end{gathered}
$$

The series in (33) is convergent for the same reason as the series in (19). The absolute value of the term with hyperbolic tangent increases approximately linearly for $-a / 2 \leq \mathrm{x} \leq \mathrm{a} / 2$ as $n$ increases while the first term is inversely proportional to the 5 th power of $n$.

Mean temperature is calculated by double integration

$$
\bar{T}=\frac{1}{a b \bar{v}} \int_{-a / 2}^{a / 2} \int_{-b / 2}^{b / 2} v(x, y) T(x, y) d x d y,
$$

which gives

$$
\begin{gathered}
\bar{T}=T_{w}+\frac{\rho c_{p}}{\lambda} \frac{d \bar{T}}{d z} \frac{1}{\mu} \frac{d p}{d z} \frac{17 d_{h}^{4}}{26880}\left(\frac{K+1}{K}\right)^{4} \times \\
\times \frac{1-\frac{40320}{17 K} \sum_{n=1}^{\infty} \frac{1}{u_{n}^{9}}\left[15 \tanh \left(t_{n}\right)-\frac{7 t_{n}+2 t_{n}^{2} \tanh \left(t_{n}\right)}{\cosh ^{2}\left(t_{n}\right)}\right]}{1-\frac{192}{K} \sum_{n=1}^{\infty} \frac{1}{u_{n}^{5}} \tanh \left(t_{n}\right)},
\end{gathered}
$$


where

$$
t_{n}=u_{n} K \frac{1}{2} .
$$

Combination of (22), (30), (35) gives the final formula for Nusselt number

$$
\mathrm{Nu}=\frac{140}{17}\left(\frac{K}{K+1}\right)^{2} \frac{\left[1-\frac{192}{K} \sum_{n=1}^{\infty} \frac{1}{u_{n}^{5}} \tanh \left(t_{n}\right)\right]^{2}}{1-\frac{40320}{17 K} \sum_{n=1}^{\infty} \frac{1}{u_{n}^{9}}\left[15 \tanh \left(t_{n}\right)-\frac{7 t_{n}+2 t_{n}^{2} \tanh \left(t_{n}\right)}{\cosh ^{2}\left(t_{n}\right)}\right]} .
$$

The solution for Nusselt number of fully developed laminar flow depends only on the wall heat transfer boundary condition (here H1) and the channel geometry represented by K [3]. Both series in (37) are rapidly convergent. In practice, for $1 \leq K \leq 20$ achievement of the relative accuracy $10^{-6}$ demands use of maximum 9 and 3, respectively, first values of the series in the numerator and the denominator.

\section{Comparison of the results}

Calculation results both of Fanning friction factor (23) and Nusselt number (37) were compared to the results of the approximate formulae from [1] (also discussed in [3-5]). The calculation using the derived exact formulae was performed by the computer program written in Object Pascal (Delphi 7 Environment). This program was used to calculate $f, \mathrm{Nu}$, temperature profiles and heat transfer in minichannel heat exchangers.

The approximate formula for Fanning friction factor has the form [1]

$$
\begin{aligned}
f \operatorname{Re} & =24\left(1-1.3553 K^{-1}+\right. \\
& \left.+1.9467 K^{-2}-1.7012 K^{-3}+0.9564 K^{-4}-0.2537 K^{-5}\right) .
\end{aligned}
$$

The results of numerical calculation of $f$ using (23), (38) are shown in Tab. 1. For comparison with circular channels, the value of the aspect ratio for $f \operatorname{Re}=16$ is also calculated. Figure 5 shows the impact of the aspect ratio on the Fanning friction factor.

Use of the value for circular cross-section instead of the actual values would create inaccuracies:

$$
\frac{f_{\text {circle }}}{f_{\text {square }}}=\frac{16}{14.23}=1.12, \quad \frac{f_{\text {circle }}}{f_{\text {plates }}}=\frac{16}{24}=0.67 .
$$


Table 1: Comparison of the exact and approximate values of Fanning friction factor.

\begin{tabular}{|c|c|c|}
\hline $\begin{array}{c}K=a / b \\
{[-]}\end{array}$ & $\begin{array}{c}f \operatorname{Re} \\
{[-]}\end{array}$ & $\begin{array}{c}f \operatorname{Re}[1] \\
{[-]}\end{array}$ \\
\hline \hline 1 & 14.23 & 14.23 \\
\hline 2 & 15.55 & 15.56 \\
\hline 2.269327 & 16.00 & 16.01 \\
\hline 3 & 17.09 & 17.09 \\
\hline 4 & 18.23 & 18.23 \\
\hline 5 & 19.07 & 19.07 \\
\hline 10 & 21.17 & 21.18 \\
\hline$\rightarrow \infty$ & 24.00 & 24.00 \\
\hline
\end{tabular}

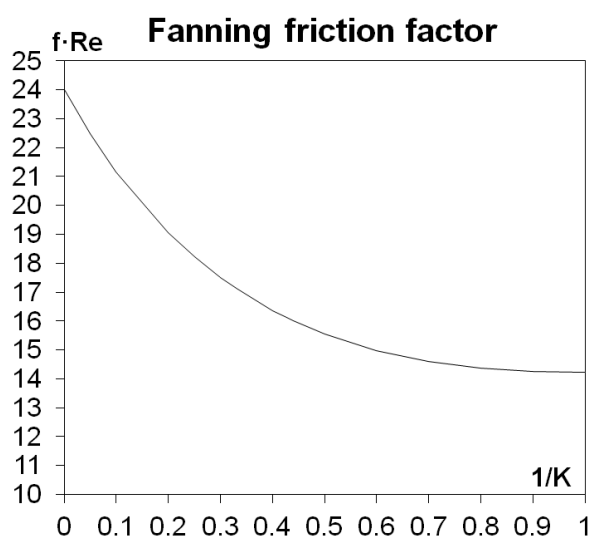

Figure 5: The impact of the cross-section aspect ratio on Fanning friction factor.

The value of $f$ would be overestimated by $12 \%$ and underestimated by $33 \%$ respectively.

The approximate formula for Nusselt number [1] has the form

$$
\begin{aligned}
\mathrm{Nu} & =8.235\left(1-2.0421 K^{-1}+\right. \\
& \left.+3.0853 K^{-2}-2.4765 K^{-3}+1.0578 K^{-4}-0.1861 K^{-5}\right) .
\end{aligned}
$$

The results of numerical calculation of $N u$ using (37), (40) are shown in Tab. 2. For comparison with circular channels, the value of aspect ratio for $\mathrm{Nu}=48 / 11=4.363636$ is also calculated. Figure 6 shows the impact of the aspect ratio on the Nusselt number. 
Table 2: Comparison of the exact and approximate calculation of Nusselt number.

\begin{tabular}{|c|c|c|}
\hline $\begin{array}{c}K=a / b \\
{[-]}\end{array}$ & $\begin{array}{c}\mathrm{Nu} \\
{[-]}\end{array}$ & $\begin{array}{c}\mathrm{Nu}[1] \\
{[-]}\end{array}$ \\
\hline \hline 1 & 3.61 & 3.61 \\
\hline 2 & 4.12 & 4.13 \\
\hline 2.342318 & 4.363636 & 4.37 \\
\hline 3 & 4.79 & 4.80 \\
\hline 4 & 5.33 & 5.33 \\
\hline 5 & 5.74 & 5.74 \\
\hline 10 & 6.78 & 6.79 \\
\hline$\rightarrow \infty$ & 8.24 & 8.24 \\
\hline
\end{tabular}

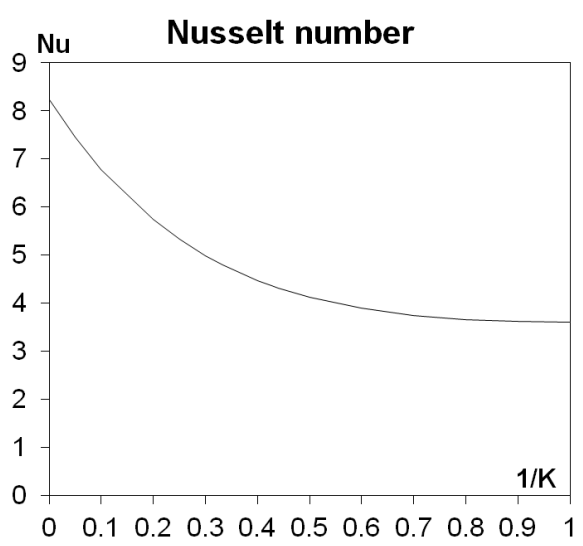

Figure 6: The impact of cross-section aspect ratio on Nusselt number.

Use of the value for circular cross-section instead of the actual value would create inaccuracies:

$$
\frac{\mathrm{Nu}_{\text {circle }}}{\mathrm{Nu}_{\text {square }}}=\frac{4.364}{3.608}=1.21, \quad \frac{\mathrm{Nu}_{\text {circle }}}{\mathrm{Nu}_{\text {plates }}}=\frac{4.364}{8.235}=0.53
$$

The value of $\mathrm{Nu}$ would be overestimated by $21 \%$ and underestimated by $47 \%$ respectively.

An example of temperature profiles simulation generated by this program is shown in Fig. 7. 


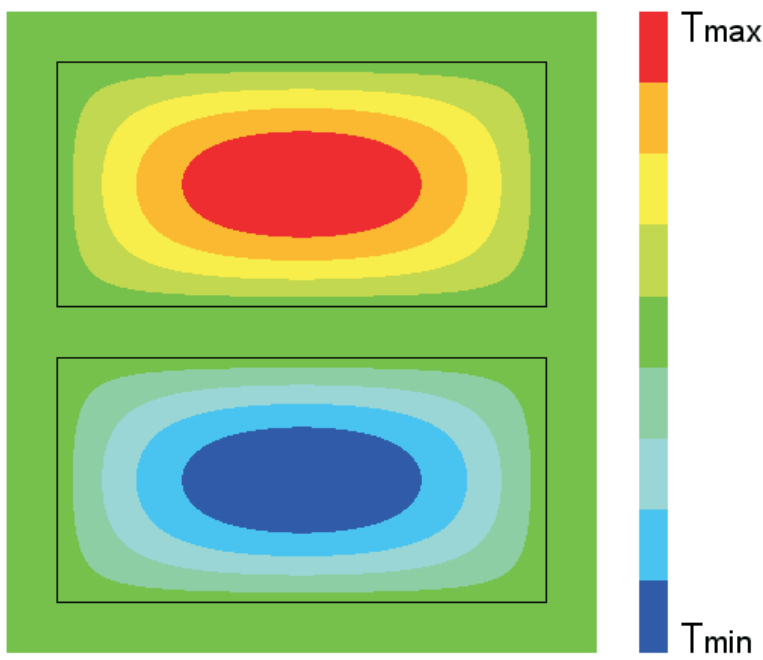

Figure 7: Simulation of temperature profiles for one pair of hot and cold laminar fluid flows in the minichannel heat exchanger (aspect ratio $K=2$ ).

\section{Conclusions}

- The exact analytical formulae in mathematically usable form for Fanning friction factor and Nusselt number were derived.

- The formula for Nusselt number contains single fast convergent infinite series instead of double series known from the literature.

- The results of obtained exact solutions and widely used approximate formulae in the literature are in very good agreement. This enables a possible use of the exact formulae instead of the approximate ones.

- Analytical form of velocity and temperature profiles allows their further adaptation to create the complete analytical model of heat transfer in minichannel heat exchangers.

Acknowledgements This work has partially been funded from a National Project POIG.01.01.02-00-016/08 Model agroenergy complexes as an example of distributed cogeneration based on a local renewable energy sources. 


\section{References}

[1] Shah R.K., London A.L.: Laminar Flow Forced Convection in Ducts. Academic Press, New York 1978.

[2] Kays W.M., Crawford M.E.: Convective Heat and Mass Transfer. McGraw-Hill, New York 1993.

[3] Kandlikar S.G., Garimella S., Li D., Colin S., King M.R.: Heat Transfer and Fluid Flow in Minichannels and Microchannels. Elsevier Ltd., Oxford 2006.

[4] Hartnett J.P., Kostic M.: Heat transfer to Newtonian and non-Newtonian fluids in rectangular ducts. Adv. Heat Transfer, 19(1989), 247-356.

[5] Papautsky I., Mohanty S., Ameel T., Frazier A.B.: Effects of rectangular microchannel aspect ratio on laminar friction constant. In: Proc. of SPIE Conference on Microfluidic Devices and Systems, Santa Clara 1999, 147-159. 